\title{
Reconstruction and exact solutions for cosmological perturbations from a generalized gravity theory.
}

\author{
José Antonio Belinchón* \\ Departamento de Matemáticas, Facultad de Ingeniería, \\ Universidad de Atacama, Avenida Copayapu 485, Copiapó, Chile. \\ Carlos Gonzáledt \\ Departamento de Física, Universidad de Atacama, \\ Avenida Copayapu 485, Copiapó, Chile. \\ Ramón Herrerat \\ Instituto de Física, Pontificia Universidad Católica de Valparaíso, \\ Avenida Brasil 2950, Casilla 4059, Valparaíso, Chile.
}

(Dated: September 6, 2019)

\begin{abstract}
Scalar and tensor cosmological perturbations during an inflationary universe scenario in the context of the a generalized gravity theory are studied. This analyze is carried out considering an ansatz on the variables associated to scalar and tensor perturbation $\left(z_{s}\right.$ and $\left.z_{t}\right)$ in the Jordan frame. In this context, we analyze two different Ansatze for the ratio $z_{s} / z_{t}$, and we study in great detail the analytical and exact solutions for the cosmological perturbations together with the corresponding reconstruction of the background variables. Recent observational data from the Planck 2018 results are employed to constrain the parameters of each of the models.
\end{abstract}

PACS numbers: $98.80 . \mathrm{Cq}$

\footnotetext{
*Electronic address: jose.belinchon@uda.cl

${ }^{\dagger}$ Electronic address: carlos.gonzalez@uda.cl

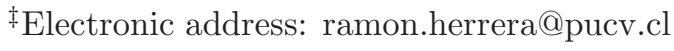




\section{INTRODUCTION}

It is well known that the inflationary stage is to date an excellent candidate for solving the long standing problems of the Big Bang model (horizon, flatness, monopoles, etc.) during the early universe [1, 2]. Nevertheless, the biggest characteristic of the inflationary universe is that it furnishes a causal interpretation to explain the observed anisotropy of the cosmic microwave background (CMB) radiation[3, 4] and also inflation gives account of the distribution of large scale structures [5].

In order to describe the inflationary epoch, the scalar field plays a fundamental role in the dynamic of the early universe. In this sense, the scalar field or inflaton can interact essentially with other fields and also with the gravitational sector. In the context of the different inflationary models that give account of the evolution of the early universe, we can distinguish those the models that use a scalar tensor theory in order to produce the accelerated expansion of the universe. The scalar to tensor theory of gravitation can be considered as an extension to the General Relativity (GR), where a scalar field couples with the gravitational sector produces a dynamic evolution on the gravitational constant or Planck mass. In this context, the scalar-tensor theory of gravity as alternative theory is the most well studied that exist in the literature. Originally, this theory was proposed in the works of Kaluza and Klein, Jordan[6], Brans and Dicke[7] and its generalization by Bergamann and Wagoner[8]. In particular, the Jordan Brans Dicke (JBD) theory is formulated by the presence of a scalar field (massless) couples with the gravitational sector from the scalar curvature applied to the cosmology. In the context of radiation problems assuming the non-minimal coupling with the Ricci scalar was analyzed in Ref.[9]. It is well known that the scalar tensor theories emerge clearly from effective theories of higher dimensional theories under a dimensional reduction, e.g. Kaluza-Klein, string[10]. In order to describe the inflationary epoch, different inflationary models have been studied in the framework of the non-minimal coupling to gravity, called the Jordan frame, see e.g. [11 13]. In relation to the Jordan and Einstein frames in Ref.[14] was analyzed a frame independent classification from the point of view of the observable parameters; the scalar spectral index

$n_{s}$ and the tensor to scalar ratio $r$, see also [15]. For a review on the scalar-tensor theory and its context in the inflationary stage, see Refs. [16-18].

On the other hand, the study of the cosmological perturbations, such as the power spec- 
trum of the scalar and tensor perturbations coming from quantum fluctuations of the vacuum in the early universe was developed in Ref.[19]. During the early universe, these fluctuations have a small amplitudes approximately of the Planck scale, and product of the accelerated expansion of the universe, these fluctuations are amplified to scales farther galactic scales.

In this context, analytical and exact solutions from the background and perturbative equations (scalar-tensor) in the case of a standard scalar field can be obtained in the Einstein frame. A de Sitter universe is an example of this, here a constant potential associated to scalar field is obtained from the background[1], and an exact results can be found from the perturbative equations for the scalar and tensor power spectrums [19]. Another example corresponds to an expansion power-law or power-law inflation. For the case of the power-law we can found an exact results for the background equations giving as result an exponential potential[20] and we can also got an exact calculation for the cosmological perturbations, see Ref.[21]. In this sense, the reconstruction of the exact and analytical background variables from the analysis of the perturbative equations of the cosmological perturbations has been considered by several authors [22]. In particular considering the Einstein framework, an exact reconstruction of the background can be obtained in the specific case in which the effective potential[22] of perturbative equations is zero (called the Easther model) [23]. However, this condition on the effective potential gives rise to a scalar spectral index $n_{S} \equiv 3$ (blue tilt), and this value is disapproved by the observational data. By considering this idea, the authors in ref. [24] consider an anzatz for the effective potential of the scalar perturbative equation in terms of the conformal time assuming a standard scalar field. Here, analytical solutions were found for the scalar and tensor power spectrums, however, the reconstruction of background (inflaton potential) was obtained numerically. Similarly, assuming an extended ansatz for the effective potential of the scalar perturbative equation in the framework of a tachyon field the authors in ref.[25] found analytical expressions for the scalar and tensor power spectrums, considering that the tachyon field slowly changes with the time during inflation. Here, the model is well supported by the Planck data only if the effective speed of sound $c_{s}$ associated the tachyonic field is $c_{s} \simeq 1$ and the effective potential of the scalar perturbative equation is similar to the power-law [25]. In this line, some approximations to the cosmological perturbations in order to obtain analytical expressions were developed in Refs. [26, 27] (see also ref.[28] for the reconstruction during the present acceleration). Also, in order to solve the scalar perturbative equation in the Einstein frame, 
the authors in Ref.[29] developed a new formalism, in which the considered methodology corresponds to Green's function method to calculate the power spectrum of the curvature perturbations produced during inflation, see also [30].

In the following, we analyze the possibility to reconstruct exactly the background variables and scalar and tensor power spectrums in the frame of a generalized scalar to tensor theory. We propound this possibility considering a new methodology from an ansatz between the scalar and tensor variables $\left(z_{s}\right.$ and $\left.z_{t}\right)$ in terms of the conformal time. In this form, we study how this relation influences the dynamics of the scalar and tensor perturbations and the background variables (scale factor, scalar potential and coupling function) in a generalized scalar to tensor theory. Since our intention is to reconstruct exactly the background variables starting from an already exact solution for cosmological perturbations, we will see that it is not necessary to consider slow roll assumption into our treatment. Also, we will explorer the parameter spaces from the cosmological perturbations and how these parameters are constrained from the observational data[31].

The outline of the paper goes as follow: In the sections II y III we give a brief description of the background equations and the cosmological perturbations in a generalized scalar to tensor theory of gravitation. In the section IV, we study a specify case in which the scalar and tensor variables are proportional (model I) and as an example we consider the power law expansion. In the section $\mathrm{V}$, we analyze an explicit expressions for the scalar and tensor variables as function of the conformal time (model II), in order to reconstruct the background and power spectrums quantities. Here, we confine the parameter spaces from Planck 2018 data. In section VI exhibits our results and conclusions. We chose units so that $c=\hbar=8 \pi G=1$.

\section{BACKGROUND EQUATIONS}

In this section we give a brief description of the background equations, considering a generalized scalar tensor theory. In this context, we start with the effective action for the scalar-tensor gravitational model in the Jordan frame given by [32]

$$
S=\int d^{4} x \sqrt{-g}\left[\frac{F(\varphi)}{2} R+\frac{\omega(\varphi)}{2} g^{\mu \nu} \varphi_{; \mu} \varphi_{; \nu}-V(\varphi)\right],
$$


where the quantity $F(\varphi)$ corresponds to arbitrary function that describes the coupling between the scalar field $\varphi$ and the geometry from the Ricci scalar $R, \omega(\varphi)$ denotes a generic function coupling the scalar field to its kinetic energy density and $V(\varphi)$ corresponds to the effective potential associated to the scalar field.

By considering the variation of the effective action given by Eq.(11) with respect to the two fields $g_{\mu \nu}$ and $\varphi$, we obtain the background equations. Let us introduce into the action (11) a spatially flat Friedmann-Lemaitre-Robertson-Walker (FLRW) metric, defined as

$$
d s^{2}=d t^{2}-a^{2}(t) d x^{i} d x_{i}=a^{2}(\eta)\left[d \eta^{2}-d x^{i} d x_{i}\right]
$$

where the new time $\eta$ denotes the conformal time defined as $d t=a d \eta$, the quantities $d x^{i} d x_{i}$ and $a(t)$ correspond to the flat three-surface and scale factor, respectively.

By assuming a spatially homogeneous scalar field $\varphi(\eta)$, then the corresponding Friedmann equation reads

$$
3 \mathcal{H}^{2}=\frac{\omega \varphi^{\prime 2}}{2 F}+\frac{a^{2} V}{F}-3 \mathcal{H} \frac{F^{\prime}}{F}
$$

and the Raychaudhuri equation for this theory can be written as

$$
\mathcal{H}^{2}-\mathcal{H}^{\prime}=\frac{\omega \varphi^{\prime 2}}{2 F}+\frac{F^{\prime \prime}}{2 F}-\frac{\mathcal{H} F^{\prime}}{F} .
$$

Here the Hubble rate in the conformal time is defined as $\mathcal{H} \equiv a^{\prime} / a$ and the scalar Ricci from metric (2) is given by $R=\frac{6}{a^{2}}\left(\mathcal{H}^{2}+\mathcal{H}^{\prime}\right)$. In the following, the prime ' denotes the derivative with respect to conformal time $\eta$.

The dynamical equation of motion for the scalar field from action (11) is given by

$$
\frac{1}{2 \omega}\left(a^{2} F_{, \varphi} R-\omega_{, \varphi} \varphi^{\prime 2}-2 a^{2} V_{, \varphi}\right)-2 \mathcal{H} \varphi^{\prime}-\varphi^{\prime \prime}=0,
$$

where the subscript $\left.{ }_{, \varphi}\right)$ denotes the derivative with respect to scalar field $\varphi$.

By combining Eqs.(31) and (44) we find that the effective potential can be rewritten as

$$
V=\frac{1}{a^{2}}\left[F\left(\mathcal{H}^{\prime}+2 \mathcal{H}^{2}\right)+2 \mathcal{H} F^{\prime}+\frac{F^{\prime \prime}}{2}\right] .
$$

Here, we note that the effective potential can be obtained in terms of the time (or scalar field) once known the scale factor and the arbitrary function $F$. 


\section{PERTURBATIONS}

In this section we will analyze the scalar and tensor perturbations for the case of the generalized scalar tensor theory. We consider that the general metric perturbations is given by

$$
d s^{2}=a^{2}(\eta)\left[(1+\Phi) d \eta^{2}-2 \partial_{i} B d x^{i} d \eta-\left[(1-2 \Psi) \delta_{i j}+2 \partial_{i} \partial_{j} E\right] d x^{i} d x^{j}\right] .
$$

From this metric and in order to describe the scalar-type structure we need the four variables $\Phi, B, \Psi$ and $E$, which are functions of the time and space coordinates [33].

On the other hand, we also consider that the perturbation associated to the field $\varphi(t, \vec{x})$ is given by $\varphi(t, \vec{x})=\varphi(t)+\delta \varphi(t, \vec{x})$, where $\varphi(t)$ is the homogeneous background field satisfying (5) and $\delta \varphi(t, \vec{x})$ corresponds to the perturbation in space and time of the scalar field.

Introducing the intrinsic curvature perturbation $\mathcal{R}$ of the comoving hypersurfaces, that gives account of the scalar perturbations defined by

$$
\mathcal{R} \equiv \Psi+\mathcal{H} \frac{\delta \varphi}{\varphi^{\prime}}
$$

then considering this intrinsic curvature the first order perturbed equations can be compacted to [34]

$$
\frac{1}{a^{3} Q_{s}} \frac{d}{d t}\left(a^{3} Q_{s} \dot{\mathcal{R}}\right)+\frac{k^{2}}{a^{2}} \mathcal{R}=0
$$

where the function $Q_{s}$ associated to the scalar perturbation in the Jordan frame is defined as $[34]$

$$
Q_{s}=\frac{\frac{3 F^{\prime 2}}{2 F}+\omega \varphi^{\prime 2}}{\left(\mathcal{H}+\frac{F^{\prime}}{2 F}\right)^{2}} .
$$

Other important quantity to describe the perturbations is the gauge-invariant potential $v$, that relates the background variables with the intrinsic curvature and is defined as $v=z_{s} \mathcal{R}$ or in the Fourier modes $v_{k}=z_{s} \mathcal{R}_{k}$. Here $k=|\boldsymbol{k}|$ denotes the modulus of the wavenumber. In this context, the new variable $z_{s}$ associated to scalar perturbations in the framework of the scalar tensor theory is defined as [34]

$$
z_{s}=a \sqrt{Q_{s}}
$$

In this form, the first order perturbed equation (9) considering the Fourier modes $v_{k}$ can be rewritten as [34, 35$]$

$$
v_{k}^{\prime \prime}+\left(k^{2}-\frac{z_{s}^{\prime \prime}}{z_{s}}\right) v_{k}=0 .
$$


We mentioned that the asymptotic limits for the Fourier modes $v_{k}$ given by Eq.(12) are determined from the a small or large scale limit. In this sense, when the conformal time $\eta$ going to $\eta \rightarrow-\infty$ corresponds to a small scale limit, in which the modes are inside the horizon. On the contrary when the conformal time $\eta \rightarrow 0$ represents to a large scale and then the modes to be outside the horizon. We also need to satisfy the Wronskian condition in which $v_{k}^{*} v_{k}^{\prime}-v_{k} v_{k}^{*^{\prime}}=-i$, in order to guarantee the commutation relations from the quantum theory $[34]$.

Under this formalism the curvature perturbation in terms of Fourier series is defined as

$$
\mathcal{R}=\int \frac{d^{3} \boldsymbol{k}}{(2 \pi)^{3 / 2}} \mathcal{R}_{\boldsymbol{k}}(\eta) e^{i \boldsymbol{k} \boldsymbol{x}}
$$

and the vacuum expectation value of $\mathcal{R}_{\boldsymbol{k}}$ is given by $\left\langle\mathcal{R}_{\boldsymbol{k}} \mathcal{R}_{\boldsymbol{l}}^{*}\right\rangle=\frac{2 \pi}{k^{3}} \mathcal{P}_{R} \delta^{3}(\boldsymbol{k}-\boldsymbol{l})$, where $\mathcal{P}_{R}(k)$ corresponds to the power spectrum. In this way, the power spectrum of the curvature perturbations is defined as

$$
\mathcal{P}_{R}(k)=\frac{k^{3}}{2 \pi^{2}}\left|\frac{v_{k}}{z_{s}}\right|^{2} .
$$

By knowing the power spectrum of the curvature perturbations, then the scalar index $n_{s}$ is given by

$$
n_{s}-1=\frac{d \ln \mathcal{P}_{R}}{d \ln k}
$$

On the other hand, it is well known that the production of tensor perturbation during the inflationary epoch would generate gravitational waves. The framework to analyze the quantum fluctuations in the gravitational field is similar to the case of the scalar perturbation developed above. From metric, the tensor perturbations are characterized by the tensor $h_{i j}$ and the equation for the Fourier modes $u_{k}$ from the analysis perturbative is similar to Eq.(12) and this equation is given by

$$
u_{k}^{\prime \prime}+\left(k^{2}-\frac{z_{t}^{\prime \prime}}{z_{t}}\right) u_{k}=0,
$$

where now the new variable $z_{t}$ associated to the modes of the tensor perturbations in the framework of the scalar tensor theory is defined from the replacement of the scalar function $Q_{s} \rightarrow Q_{t}=F[34, \underline{35}]$ such that

$$
z_{t}=a \sqrt{Q_{t}}=a \sqrt{F}
$$


In analogy to the scalar perturbations, the power spectrum of the tensor perturbations as function of the modes $v_{k}$, is denoted as

$$
\mathcal{P}_{g}=\frac{2 k^{3}}{\pi^{2}}\left|\frac{u_{k}}{z_{t}}\right|^{2}
$$

where now the tensor spectral index $n_{T}$ is defined as $n_{T}=d \ln \mathcal{P}_{g} / d \ln k$.

In the cosmological context, a fundamental observational quantity is related to the tensor to scalar ratio $r$, which is defined as

$$
r=\frac{\mathcal{P}_{g}}{\mathcal{P}_{R}} .
$$

Observationally, the BICEP2/Keck-Array collaboration [36] published the upper bound on the tensor-to-scalar ratio to be $r<0.07$ (95\% CL). Recently, the Planck 2018 results [31] made it known that when the likelihood is combined with B-mode polarization of BICEP2/Keck-Array collaboration the ratio $r<0.064$ (95\% CL).

The equations for the respective modes (12) and (16), may be considered as a time independent Schrödinger equation of the type $f_{k}^{\prime \prime}+\left(k^{2}-U\right) f_{k}=0$, where the effective potential as function of the conformal time is defined as $U=U(\eta)=z_{s}^{\prime \prime} / z_{s}$ for the scalar modes in which $f_{k}=v_{k}$ and $U=U(\eta)=z_{t}^{\prime \prime} / z_{t}$ for the tensor modes $f_{k}=u_{k}$, respectively.

As we can observe from (12) and (16), the evolution of the scalar and tensor cosmological perturbations during the inflationary epoch, are determined by the functions $z_{s}(\eta)$ and $z_{t}(\eta)$, however these functions at the same time are related to the effective potential of cosmological perturbations $U(\eta)$.

A methodology used in the literature in order to find analytical solutions to the modes $v_{k}$ and $u_{k}$ is to consider an "ansatz" for the effective potential of the cosmological perturbations $U(\eta)$, achieving thus the reconstruction of the background variables, such as the scalar potential $V(\phi)$ and the scale factor $a(t)$ analytically (or numerically). In particular in the framework of the GR and considering a standard scalar field analytical solutions can be obtained in order to reconstruct the background variables (such as effective potential $V(\phi))$. In particular we have; de Sitter inflation in which the effective potential of the scalar perturbation is given by $U(\eta)=\frac{2}{\eta^{2}}$ giving a potential $V(\phi)=$ constant [22], the expansion power law inflation $\left(a \sim t^{p}\right.$ with $\left.p>1\right)$ where the effective potential of the scalar perturbation results $U(\eta)=\frac{\nu^{2}-1 / 4}{\eta^{2}}$, where $\nu$ is a constant defined as $\nu=3 / 2+1 /(p-1)$ and the reconstruction gives origin to an exponential potential associated to scalar field [21]. Also, considering the ansatz for the effective potential $U(\eta)=C_{1} / \eta+C_{2} / \eta^{2}$, where $C_{1}$ and 
$C_{2}$ are constants was studied in ref.[24] and the case in which $U(\eta)=0$ or equivalently $z_{s}=0$ was developed in [23]. In the context of a tachyon field, the effective potential for the scalar perturbations $U(\eta)=0$ together with the ansatz $U(\eta)=C_{0}+C_{1} / \eta+C_{2} / \eta^{2}$, were analyzed in ref.[25]. Here, we mentioned that the reconstruction of the scalar potential associated to the tachyonic field $V(\phi)$ for the ansatz $U(\eta)=C_{0}+C_{1} / \eta+C_{2} / \eta^{2}$ was obtained numerically.

In this sense, the evolution of the scalar and tensor cosmological perturbations during the inflationary epoch are completely determined from the functions $z_{s}$ and $z_{t}$. In the following, we will develop a different methodology to obtain the reconstruction of the background from the an ansatz for the potential of the cosmological perturbations $U(\eta)$. Instead, we shall find a relation (differential equation) between the functions $z_{s}$ and $z_{t}$. In fact, combining Eqs.(44) and (10), we can eliminate the term $\omega \varphi^{\prime 2}$, then using the definitions of the functions $z_{s}$ and $z_{t}$ given by Eqs.(11) and (17), we obtain that the relation between these variables is given by

$$
z_{s}^{2}=2 z_{t}^{2}\left[1+\left(\frac{z_{t}}{z_{t}^{\prime}}\right)^{\prime}\right] .
$$

In the following, we will study different solutions from the relation given by Eq.(20). From these solutions we will find the background variables and its reconstruction in the Jordan frame as also the cosmological perturbations.

\section{MODEL I: $z_{s} \propto z_{t}$}

A particular relation between the variables $z_{s}$ and $z_{t}$ that satisfies Eq.(20), is given by $z_{s}=\gamma z_{t}$, in which $\gamma$ denotes a constant. We mention that this relation between $z_{s}$ and $z_{t}$ i.e., $z_{s} \propto z_{t}$ is equivalent to consider the slow- roll approximation in the Einstein gravity limit. In order to obtain this limit, we have that the function $F(\varphi)=\frac{1}{8 \pi G}=$ constant, and the parameter $\omega=1$, then Eq.(10) can be written as $Q_{s}=\frac{\epsilon}{4 \pi G}$, where $\epsilon$ is the usual slow roll parameter defined as $\epsilon=1-\frac{\mathcal{H}^{\prime}}{\mathcal{H}^{2}}$. By assuming that during the slow-roll regime, the parameter $\epsilon \simeq$ constant [35], and combining Eqs.(11) and (17), we get that $z_{s}=\gamma z_{t}$ in which we identify $\gamma=\sqrt{2 \epsilon}$.

From the relation in which $z_{s} \propto z_{t}$, we find a particular solution to Eq.(20) given by

$$
z_{s} \propto z_{t} \propto \eta^{q}
$$

where the power $q=\left(\frac{\gamma^{2}}{2}-1\right)^{-1}=$ constant with $\gamma \neq \pm \sqrt{2}$. Note that in the particular case 
in which $\gamma= \pm \sqrt{2}$, we have the solution $z_{s} \propto z_{t} \propto e^{c \eta}$, in which $c=$ constant.

From the particular solution given by Eq.(21) and considering of the variable $z_{t}$ (see Eq.(17)), we find that the arbitrary function $F$ becomes

$$
F \propto \frac{\eta^{2 q}}{a^{2}}
$$

In this form, in order to reconstruct the function $F$ in terms of the scalar field $\varphi$ from Eq.(22), we have to give the scale factor $a$ in terms of the time $t$ (or $\eta$ ).

On the other hand, the cosmological perturbations in the case in which $z_{s} \propto z_{t}$ can be determined, considering the particular solution given by Eq.(21). In this respect, from Eqs.(12) and (16), the solutions for both modes i.e., scalar and tensor modes in the largescale limit are given by [35]

$$
v_{k}(\eta)=u_{k}(\eta) \simeq-\frac{\sqrt{\pi \eta}}{2} \frac{i}{\pi} \Gamma(\nu)\left(\frac{k|\eta|}{2}\right)^{-\nu},
$$

where the power $\nu$ is defined as $\nu \equiv \sqrt{q(q-1)+1 / 4}$. Here, we mention that this solution for $v_{k}\left(\right.$ or $\left.u_{k}\right)$ is independent of the scale factor $a(t)$ how can it be seen of Eq.(21). From Eq.(23), we find that the spectral index and tensor-to-scalar ratio are given by [35]

$$
n_{s}=4-\sqrt{4 q(q-1)+1}
$$

and

$$
r=16(1+1 / q)
$$

Note that these results are equivalent to those obtained during power- law inflation in Einstein frame. The reason is because the function $F$ connects the conformal transformation between both frames. Thus, from Eq.(22) obtained under the particular solution $z_{s} \propto z_{t}$ and considering the conformal transformation $\hat{a}(\hat{t})=a(t) \sqrt{F}$ and $d \hat{t}=d t \sqrt{F}$, see [35], 37], we have $\hat{a}(\hat{t}) \propto \hat{t}^{\frac{q}{1+q}}$ i.e., power law inflation in the Einstein frame. [†]. In this point we emphasize that we have not assumed slow roll approximation[§] and as we mentioned before the explicit function for the scale factor $a(t)$ does not has been specified yet.

[ $\ddagger$ ] Here the caret means quantity measured in Einstein frame.

[§] Indeed, starting from Eqs. (11) and (17) and one finds [35] $\left.\frac{z_{s, t}^{\prime \prime}}{z_{s, t}}=\mathcal{H}^{2}\left\{\left(1+\delta_{s, t}\right)\left(2-\epsilon+\delta_{s, t}\right)+\frac{\delta_{s, t}^{\prime}}{\mathcal{H}}\right)\right\}$, where $\delta_{s, t}=\frac{\dot{Q}_{s}}{2 \mathcal{H} Q} \equiv \delta_{s}$ for scalar perturbations and $\delta_{s, t}=\frac{F}{2 \mathcal{H F}} \equiv \delta_{t}$ for tensor one. In the slow roll approximation, $\epsilon$ and $\delta_{s, t}$ are supposed to be constant during inflation stage, hence $\frac{z_{s, t}^{\prime \prime}}{z_{s, t}}=\frac{\gamma_{s, t}}{\eta^{2}}$ with 
From the observational point of view, we have that from the Planck data results, the scalar spectral index $n_{s} \simeq 0.964$ and the tensor to scalar ratio $r<0.1[31]$. From these observational data, we find that the parameter $q=\left(\gamma^{2} / 2-1\right)^{-1}$ takes two values; $q=-1.02$ and $q=2.02$, by considering that the scalar spectral $n_{s}=0.94$. Thus, evaluating the tensor to scalar ratio $r$ given by Eq.(25), we have that for the value $q=-1.02$ corresponds to $r \simeq 0.3$ and for the value $q=2.02$ the parameter $r \simeq 24$. In this form, we show that the model is not well supported by the observational data, with which the solution $z_{s} \propto z_{t}$ does not work.

Although the model $z_{s} \propto z_{t}$ does not work, we consider an example to reconstruct the background variables (scalar potential $V(\varphi)$ and coupling function $F(\varphi)$ )for a specific inflationary model. In particular, we consider the simplest situation in which the parameter $w=1$ or induced gravity and the inflationary expansion with the scale factor $a(t) \propto t^{p}$ ( or equivalently $a \propto \eta^{\frac{p}{1-p}}$ ) with $p>1$ (power-law inflation). Thus, considering Eq.(22) we can integrate Eq. (4), obtaining that the scalar field $\varphi$ as function of the conformal time results

$$
\varphi(\eta) \propto \eta^{-\frac{p}{1-p}+q_{1}}
$$

with $q_{1}=$ constant such that $q_{1} \neq \frac{p}{1-p},-1$.

In this way, replacing Eq.(26) in Eq.(22), the reconstruction of the function $F(\varphi)$ during the power law inflation takes the form

$$
F(\varphi)=\alpha^{-2} \varphi^{2} \equiv \xi \varphi^{2}
$$

where the constant $\alpha$ is defined as $\alpha=\frac{c}{-\frac{p}{1-p}+q_{1}}$, in which $c=\sqrt{-6\left(\frac{p}{1-p}-q_{1}\right)^{2}+2 q\left(1+q_{1}\right)}=$ $\sqrt{\triangle} \neq 0$ and $\triangle>0$ such that $c \in \mathbb{R}$. Here, we can identify the constant $\alpha^{-2}=\xi$, wherewith corresponding to the theory of the Induced Gravity with the specific function $F(\varphi)$ given by Eq.(27) in which $\xi>0$, see Refs. [38, 39].

By considering Eq.(6), we find that the reconstruction of the effective potential $V(\varphi)$ during the stage of power law inflation becomes

$$
V(\varphi) \propto \varphi^{2 \beta}
$$

the constant $\gamma_{s, t}=\frac{\left(1+\delta_{s, t}\right)\left(2-\epsilon+\delta_{s, t}\right)}{(1-\epsilon)^{2}}$. In contrast, from Eqs.(11), (21) and (22), we have $Q_{s} \propto F$, hence $\delta_{s, t}=\frac{q}{\mathcal{H} \eta}-1$ and, as usual, $\epsilon=1-\frac{\mathcal{H}^{\prime}}{\mathcal{H}^{2}}$. Thus, we have $\frac{z_{s, t}^{\prime \prime}}{z_{s, t}}=\frac{q(q-1)}{\eta^{2}}$. As we can see, there is no need to assume slow roll approximation. 
where the power $\beta$ is defined as $\beta=1-\frac{1}{q-p\left(1+q_{1}\right)}$.

\section{MODEL II: $z_{s}=z_{t} f(\eta)$}

In this section we develop a specific ansatz for the ratio between variables $z_{t}$ and $z_{s}$ in terms of the conformal time $\eta$, in order to obtain a solution to Eq.(20). In this context, we assume that the relation between the functions $z_{s}$ and $z_{t}$ is given by

$$
z_{s}=z_{t} f(\eta)
$$

where $f(\eta)$ is a function of the conformal time $\eta$. Note that for the model I studied previously, we have the specific case in which the function $f(\eta)=$ constant, such that $z_{t} \propto z_{s}$.

By replacing Eq.(29) in Eq.(20) we find that the function $f(\eta)$ is related with the tensor variable $z_{t}$ as

$$
f(\eta)^{2}=2\left[1+\left(\frac{z_{t}}{z_{t}^{\prime}}\right)^{\prime}\right]=2\left[1+\left(\frac{1}{\left(\ln z_{t}\right)^{\prime}}\right)^{\prime}\right] .
$$

In this form, we find that the general solution for the tensor function $z_{t}$ in terms of the $f(\eta)$ can be written as

$$
z_{t}(\eta)=\exp \left[\int\left(\int\left[\frac{f(\eta)^{2}}{2}-1\right] d \eta\right)^{-1} d \eta\right] .
$$

Thus, we can give a specific function or ansatz for $f$ is terms of the conformal time, in order to obtain the tensor function $z_{t}$.

An interesting situation occurs when we choose that the function $f(\eta)$ is given by the ansatz

$$
f(\eta)=\sqrt{2}\left[1+\frac{\beta_{1}}{\left(\beta_{1}-\alpha_{1}|\eta|\right)^{2}}\right]^{1 / 2},
$$

where $\beta_{1}$ and $\alpha_{1}$ are constants. The constant $\beta_{1}$ is a dimensionless constant and $\alpha_{1}$ has dimensions of mass.

In this sense, replacing Eq.(32) in (31), we find that a particular solution for the tensor function $z_{t}$ can be written as

$$
z_{t}(\eta) \propto|\eta|^{\beta_{1}} e^{-\alpha_{1}|\eta|}
$$

Thus, from solution (33), we obtain that the effective potential $U(\eta)$ of the tensor perturbation becomes

$$
U(\eta)=\frac{z_{t}^{\prime \prime}}{z_{t}}=C_{0}^{t}+\frac{C_{1}^{t}}{|\eta|}+\frac{C_{2}^{t}}{\eta^{2}}
$$


where the constants $C_{0}^{t}, C_{1}^{t}$ and $C_{2}^{t}$ are defined as

$$
C_{0}^{t}=\alpha_{1}^{2}, \quad C_{1}^{t}=-2 \alpha_{1} \beta_{1}, \quad \text { and } \quad C_{2}^{t}=\beta_{1}\left(\beta_{1}-1\right),
$$

respectively. Then, the constant $C_{0}^{t}$ has dimensions of mass squared, $C_{1}^{t}$ has dimensions of mass and $C_{2}^{t}$ is a dimensionless constant. Also, we note that the constant $C_{1}^{t}$ depends on the constants $C_{0}^{t}$ and $C_{2}^{t}$, respectively.

From Eq.(34) we note that a general solution for the tensor variable $z_{t}$ can be written as

$$
z_{t}(\bar{\eta})=A_{1}^{t} W_{\alpha_{t}, \beta}(\bar{\eta})+A_{2}^{t} W_{-\alpha_{t}, \beta}(-\bar{\eta})
$$

where the function $W_{ \pm \alpha_{t}, \beta}$ corresponds to the Whittaker function [40] and $A_{1}^{t}$ together with $A_{2}^{t}$ are two integration constants. Note that for the solution (35), we have used the change of the variables given by

$$
\bar{\eta}=2 \sqrt{C_{0}^{t}}|\eta|=2 \alpha_{1}|\eta|, \alpha_{t}=-\frac{C_{1}^{t}}{2 \sqrt{C_{0}^{t}}}=\beta_{1}, \text { and } \quad \beta^{2}=\frac{1}{4}+C_{2}^{t}=\frac{1}{4}+\beta_{1}\left(\beta_{1}-1\right),
$$

respectively.

In this sense, we can recognize that the particular solution (33) corresponds to the large scale limit $(|\eta| \longmapsto 0)$, of the Whittaker function $W_{\alpha_{t}, \beta}$ defined as [40]

$$
z_{t}(\eta) \rightarrow B_{t}\left(2 \sqrt{C_{0}^{t}}|\eta|\right)^{\frac{1}{2}-\beta} e^{-\sqrt{C_{0}^{t}}|\eta|} \propto|\eta|^{\beta_{1}} e^{-\alpha_{1}|\eta|}=C_{0}|\eta|^{\beta_{1}} e^{-\alpha_{1}|\eta|},
$$

where we can recognize to $\beta_{1}=\frac{1}{2}-\beta$ and the constant $C_{0}=B_{t}\left(2 \sqrt{C_{0}^{t}}\right)^{\frac{1}{2}-\beta}$.

In order to take the large scale limit of the Whittaker function $W_{\alpha_{t}, \beta}$ given by Eq.(35), we have defined $B_{t} \equiv A_{1}^{t} \frac{\Gamma(2 \beta)}{\Gamma\left(\frac{1}{2}+\beta-\alpha_{t}\right)}=A_{1}^{t} \frac{\Gamma(2 \beta)}{\Gamma\left(\frac{1}{2}+\beta-\beta_{1}\right)}=C_{0}\left(2 \alpha_{1}\right)^{\beta-\frac{1}{2}}$ where $\Gamma$ corresponds to the Gamma function and we also choose $A_{2}^{t}=0$ in the general solution given by Eq.(35) without loss of generality.

Thus, in relation to the spectrum of gravity waves, we note that the equation for the tensor-modes $u_{k}$ combining Eqs.(16) and (34) can be written as

$$
\frac{d^{2} u_{k}(\eta)}{d \eta^{2}}+\left(k^{2}-C_{0}^{t}-\frac{C_{1}^{t}}{|\eta|}-\frac{C_{2}^{t}}{\eta^{2}}\right) u_{k}(\eta)=0 .
$$

From Eq.(37), we find that the solution for the Fourier modes $u_{k}$ can be written as

$$
u_{k}(\tilde{\eta})=B_{1} W_{\tilde{\alpha_{t}}, \beta}(\tilde{\eta})+B_{2} W_{-\tilde{\alpha_{t}}, \beta}(-\tilde{\eta})
$$

where the conformal time $\tilde{\eta}$ is defined as $\tilde{\eta} \equiv 2 i k_{\text {eff }}^{t}|\eta|, k_{\text {eff }}^{t} \equiv \sqrt{k^{2}-C_{0}^{t}}$ and $\tilde{\alpha}_{t} \equiv \frac{C_{1}^{t} i}{2 k_{\text {eff }}^{t}}$. Also, the constants $B_{1}$ and $B_{2}$ correspond to integration constants and these are fixed by 
initial conditions. In particular for de Sitter universe in the frame of Einstein (where $F=1$ ) the solution for the tensor-modes $u_{k}=(1 / \eta)\left[B_{1}(k \eta \cos [k \eta]-\sin [k \eta])+B_{2}(k \eta \cos [k \eta]+\right.$ $\sin [k \eta])]$ where $C_{0}^{t}=0, C_{1}^{t}=0$ and $C_{2}^{t}=2$, since the scale factor $a \propto 1 /|\eta|$.

From Eqs.(35) and (38), the power spectrum of the tensor perturbation becomes

$$
\mathcal{P}_{g}=\frac{2 k^{3}}{\pi^{2}}\left|\frac{u_{k}}{z_{t}}\right|^{2}=\frac{2 k^{3}}{\left(\pi A_{1}^{t}\right)^{2}} \frac{\left|B_{1} W_{\tilde{\alpha_{t}}, \beta}(\tilde{\eta})+B_{2} W_{-\tilde{\alpha_{t}, \beta}}(-\tilde{\eta})\right|^{2}}{\left|W_{\alpha_{t}, \beta}(\bar{\eta})\right|^{2}} .
$$

In order to determine the constants $B_{1}$ and $B_{2}$, it is necessary to consider the quantities associated to the tensor modes $u_{k}\left(\eta_{i}\right)$ and $u_{k}^{\prime}\left(\eta_{i}\right)$ at initial conformal time $\eta_{i}$. Following Ref.[22], we can consider that the initial conditions for the modes $u_{k}\left(\eta_{i}\right)$ and $u_{k}^{\prime}\left(\eta_{i}\right)$ are given by

$$
u_{k}\left(\eta_{i}\right)=u_{k_{\eta_{i}}}=\left[k^{2}-C_{0}^{t}-\frac{C_{1}^{t}}{\left|\eta_{i}\right|}-\frac{C_{2}^{t}}{\eta_{i}^{2}}\right]^{-1 / 4}, \quad \text { and } \quad u_{k}^{\prime}\left(\eta_{i}\right)=\frac{i}{u_{k_{\eta_{i}}}}
$$

In this context, it is possible to obtain the values of the integration constants $B_{1}$ and $B_{2}$, considering the asymptotic conduct of the Whittaker function $W_{\tilde{\alpha_{t}}, \beta}(\tilde{\eta})$ in the small-limit $(|\eta| \rightarrow \infty)$ in which $W_{\tilde{\alpha_{t}}, \beta}(\tilde{\eta}) \rightarrow|\tilde{\eta}|^{\tilde{\alpha_{t}}} \exp \left(-i k_{\text {eff }}|\eta|\right)$ and together with the initial conditions given by Eq. (40) we have

$$
B_{1}=\frac{\tilde{\alpha}_{t}}{2 \sqrt{k_{e f f}^{t}}} \frac{\left(2 k_{e f f}^{t}\left|\eta_{i}\right|\right)^{-\frac{i C_{1}^{t}}{2 k_{e f f}^{t}}}}{\left(\tilde{\alpha}_{t}-i k_{e f f}^{t}\left|\eta_{i}\right|\right)} e^{i k_{e f f}^{t}\left|\eta_{i}\right|+\frac{\pi C_{1}^{t}}{4 k_{e f f}^{t}}}
$$

and

$$
B_{2}=\frac{1}{2 \sqrt{k_{e f f}^{t}}} \frac{\left(\tilde{\alpha}_{t}-2 i k_{e f f}^{t}\left|\eta_{i}\right|\right)}{\left(\tilde{\alpha}_{t}-i k_{e f f}^{t}\left|\eta_{i}\right|\right)}\left(-2 k_{e f f}^{t}\left|\eta_{i}\right|\right)^{\left.\frac{i C_{1}^{t}}{2 k_{e f f}^{t}}\right) e^{-\left(i k_{e f f}^{t}\left|\eta_{i}\right|+\frac{\pi C_{1}^{t}}{4 k_{e f f}^{t}}\right)}}
$$

respectively. Here, we have considered that in the limit in which $|\eta| \rightarrow \infty$ (or small-scale), the quantity $\left(k^{2}-C_{0}^{t}-C_{1}^{t} /\left|\eta_{i}\right|-C_{2}^{t} / \eta_{i}^{2}\right) \simeq\left(k^{2}-C_{0}^{t}\right)>0$.

In this way, the power spectrum of the tensor perturbation in the large scale limit (or equivalently $|\eta| \rightarrow 0$ ) from Eq.(39) becomes

$$
\begin{gathered}
\mathcal{P}_{g}(k)=\frac{2 k^{3}}{\pi^{2} A_{1}^{t 2}}\left|\Gamma\left(1 / 2+\beta-\alpha_{t}\right)\right|^{2}\left(\frac{k_{e f f}^{t 2}}{C_{0}^{t}}\right)^{1 / 2-\beta}\left[\frac{\left|B_{1}\right|^{2}}{\left|\Gamma\left(1 / 2+\beta-\tilde{\alpha}_{t}\right)\right|^{2}}+\right. \\
\left.\frac{\left|B_{2}\right|^{2}}{\left|\Gamma\left(1 / 2+\beta+\tilde{\alpha}_{t}\right)\right|^{2}}+(-1)^{1 / 2-\beta} \operatorname{Re}\left(\frac{2 B_{1} B_{2}^{*}}{\Gamma\left(1 / 2+\beta-\tilde{\alpha_{t}}\right) \Gamma^{*}\left(1 / 2+\beta+\tilde{\alpha}_{t}\right)}\right)\right] .
\end{gathered}
$$


On the other hand, we find that the scalar function $z_{s}$ in the large scale limit, from Eqs.(29), (32) and (36) can be written as

$$
z_{s}(\eta) \simeq \sqrt{2} B_{t}\left(2 \alpha_{1}\right)^{\beta_{1}}|\eta|^{\beta_{1}} e^{-\alpha_{1}|\eta|}\left[1+\frac{\beta_{1}}{\left(\beta_{1}-\alpha_{1}|\eta|\right)^{2}}\right]^{1 / 2}
$$

or equivalently

$$
z_{s}(\eta)=\frac{\sqrt{2} B_{t}\left(2 \sqrt{C_{0}^{t}}|\eta|\right)^{\frac{1}{2}-\beta} e^{-\sqrt{C_{0}^{t}}|\eta|}}{(1-2 \beta)\left(2 \sqrt{C_{0}^{t}}|\eta|\right)^{-1}-1} \sqrt{1+(1-2 \beta)\left(2 \sqrt{C_{0}^{t}}|\eta|\right)^{-2}\left\{3-2 \beta-4 \sqrt{C_{0}^{t}}|\eta|\right\}} .
$$

Assuming the large scale limit in which $|\eta| \longmapsto 0$, we can expand in power series the expression $z_{s}^{\prime \prime} / z_{s}$ or the effective potential associated to the scalar function in which the first order perturbed equation (12) for the scalar modes $v_{k}$ can be written as

$$
v_{k}^{\prime \prime}(\eta)+\left(k^{2}-C_{0}^{s}-\frac{C_{1}^{s}}{|\eta|}-\frac{C_{2}^{s}}{\eta^{2}}\right) v_{k}(\eta) \simeq 0,
$$

where the constants $C_{i}^{s}$ are functions of the $C_{i}^{t}$, such that

$$
\begin{gathered}
C_{0}^{s}=\frac{81-160 \beta+152 \beta^{2}-64 \beta^{3}+16 \beta^{4}}{\left(3-8 \beta+4 \beta^{2}\right)^{2}} C_{0}^{t}, \\
C_{1}^{s}=\frac{-1-8 \beta+4 \beta^{2}}{(-3+2 \beta)} \sqrt{C_{0}^{t}}, \quad \text { and } \quad C_{2}^{s}=-\frac{1}{4}+\beta^{2}=C_{2}^{t} .
\end{gathered}
$$

Redefining the conformal time $\eta$ together with the constants $C_{0}^{s}, C_{1}^{s}$ and $C_{2}^{s}$ (or $\beta$ ) such that; $\tilde{\eta} \equiv 2 i k_{\text {eff }}^{s}|\eta|$ in which $k_{\text {eff }}^{s} \equiv \sqrt{k^{2}-C_{0}^{s}}>0, \tilde{\alpha}_{s} \equiv \frac{C_{1}^{s} i}{2 k_{\text {eff }}^{s}}$ and $C_{2}^{s}$ (see Eq.(48)), we have that the general solution of Eq.(46) for the Fourier modes $v_{k}$ is given by

$$
v_{k}(\tilde{\eta})=A_{0} W_{\tilde{\alpha_{s}, \beta}}(\tilde{\eta})+A_{1} W_{-\tilde{\alpha_{s}, \beta}}(-\tilde{\eta})
$$

in which $A_{0}$ and $A_{1}$ are two integration constants and $W_{\tilde{\alpha_{s}}, \beta}$ denotes a new Whittaker function.

On the other hand, the constants $A_{0}$ and $A_{1}$ can be determined considering that in the small scale limit in which $|\eta| \rightarrow \infty$, the Fourier modes $v_{k} \rightarrow \frac{1}{\sqrt{2 k_{e f f}^{s}}} \exp \left[-i k_{e f f}^{s} \tilde{\eta}\right]$ together with the Wronskian condition. Thus, we obtain that the integration constant $A_{0}=\frac{e^{\frac{\pi C_{1}^{s}}{4 k_{1} f f}}}{\sqrt{2 k_{e f f}^{s}}}$ and the constant $A_{1}=0$, in order to satisfy these conditions. Thus, we find that the Fourier modes $v_{k}$ can be written as

$$
v_{\kappa}(\tilde{\eta})=\frac{e^{\frac{\pi C_{1}^{s}}{4 k_{e f f}}}}{\sqrt{2 k_{e f f}^{s}}} W_{\tilde{\alpha}_{s}, \beta}(\tilde{\eta}) .
$$


In order to obtain the scalar power spectrum of the curvature perturbations, we need to consider the growing modes of the $v_{k}$. These large-modes are obtained at large scale limit of the function $v_{k}(|\eta| \rightarrow 0)$, archiving thus that the quantum fluctuations are frozen outside horizon. In this sense, considering that the behavior of the Whittaker function when $|\tilde{\eta}| \rightarrow 0$ is given by

$$
W_{\tilde{\alpha_{s}, \beta}}(\tilde{\eta}) \rightarrow \frac{\Gamma(2 \beta)}{\Gamma(s)} \tilde{\eta}^{1 / 2-\beta} e^{-\tilde{\eta} / 2} \quad \text { with } \quad s \equiv 1 / 2+\beta-\tilde{\alpha}_{s}
$$

In this way, from Eq. (50) we get that the scalar modes $v_{k}$ for large scale as function of the conformal time $\eta$ becomes

$$
v_{k}(\eta)=\frac{e^{\frac{\pi C_{1}^{s}}{4 k_{e f f}}}}{\sqrt{2 k_{e f f}^{s}}} \frac{\Gamma(2 \beta)}{\Gamma(s)}\left[2 i k_{e f f}^{s} \eta\right]^{1 / 2-\beta} e^{-i k_{e f f}^{s} \eta} .
$$

In order to obtain the scalar power spectrum $\mathcal{P}_{\mathcal{R}}(k)$, we consider that the spectrum is evaluated when the wavelength of the perturbation crosses the Hubble radius i.e., $k^{2}=z_{s}^{\prime \prime} / z_{s}$, (see e.g., the review the inflation in Ref. [41]). From Eq. (46]) we find that $z_{s}^{\prime \prime} / z_{s} \simeq C_{0}^{s}+C_{1}^{s} / \eta+C_{2}^{s} / \eta^{2}$, with which we have that the conformal time when the wavelength of the perturbation crosses the Hubble radius is given by $-\eta(k)=-\eta_{*}=$ $\left[-C_{1}^{s}+\sqrt{C_{1}^{s} 2-4 C_{0}^{s} C_{2}^{s}+4 C_{2}^{s} k^{2}}\right] /\left[2\left(k^{2}-C_{0}^{s}\right)\right]$. We note that in the special case of de Sitter inflation $C_{0}^{s}=C_{1}^{s}=0$, then $-k \eta \simeq 1$ and $k \simeq a H$.

By combining Eqs.(14) and (51), we find that scalar power spectrum $\mathcal{P}_{\mathcal{R}}(k)$ becomes

$$
\mathcal{P}_{\mathcal{R}}(k)=\left(\frac{k^{3 / 2}}{2 \pi C_{0}}\right)^{2} e^{2 \alpha_{1}\left|\eta_{*}\right|+\frac{\pi C_{1}^{s}}{2 k_{\text {eff }}^{s}}}\left[1+\frac{\beta_{1}}{\left(\beta_{1}-\alpha_{1}\left|\eta_{*}\right|\right)^{2}}\right]^{-1} \frac{\Gamma^{2}(2 \beta)}{\Gamma\left(s^{*}\right) \Gamma(s)}\left(2 k_{\text {eff }}^{s}\right)^{-2 \beta},
$$

and as before the quantity $\Gamma$ denotes the Gamma function. Now, combining Eqs.(15) and (52), we obtain that the scalar spectrum index $n_{s}$ can be written as

$$
\begin{gathered}
n_{s}(k)=1+\frac{d \ln \mathcal{P}_{\mathcal{R}}}{d \ln k}=4-\frac{k^{2}}{k_{\text {eff }}^{s}}\left[\frac{2 \beta}{k_{\text {eff }}^{s}}+\frac{C_{1}^{s}}{2 k_{\text {eff }}^{s 2}}\left(\pi+i\left[\Psi_{0}(s)-\Psi_{0}\left(s^{*}\right)\right]\right)\right]+ \\
4 k^{2} \alpha_{1}\left(k^{2}-C_{0}^{s}\right)^{-1}\left[\left|\eta_{*}\right|+\frac{C_{2}^{s}}{C_{1}^{s}-2\left|\eta_{*}\right|\left(k^{2}-C_{0}^{s}\right)}\right]\left[\frac{\beta_{1}\left(\beta_{1}-\alpha_{1}\left|\eta_{*}\right|\right)^{-1}}{\beta_{1}+\left(\beta_{1}-\alpha_{1}\left|\eta_{*}\right|\right)^{-2}}-1\right],
\end{gathered}
$$

where the quantity $\Psi_{0}(s)$ corresponds to the Polygamma function.

In Fig:1 we show the tensor to scalar ratio $r$ versus the scalar spectral index $n_{s}$, for three different values of the parameter $A_{1}^{t}$ for the model II, by assuming the ansatz given by Eq.(32) for the function $f(\eta)$. In this panel we use the two-marginalized constraints for the relation 


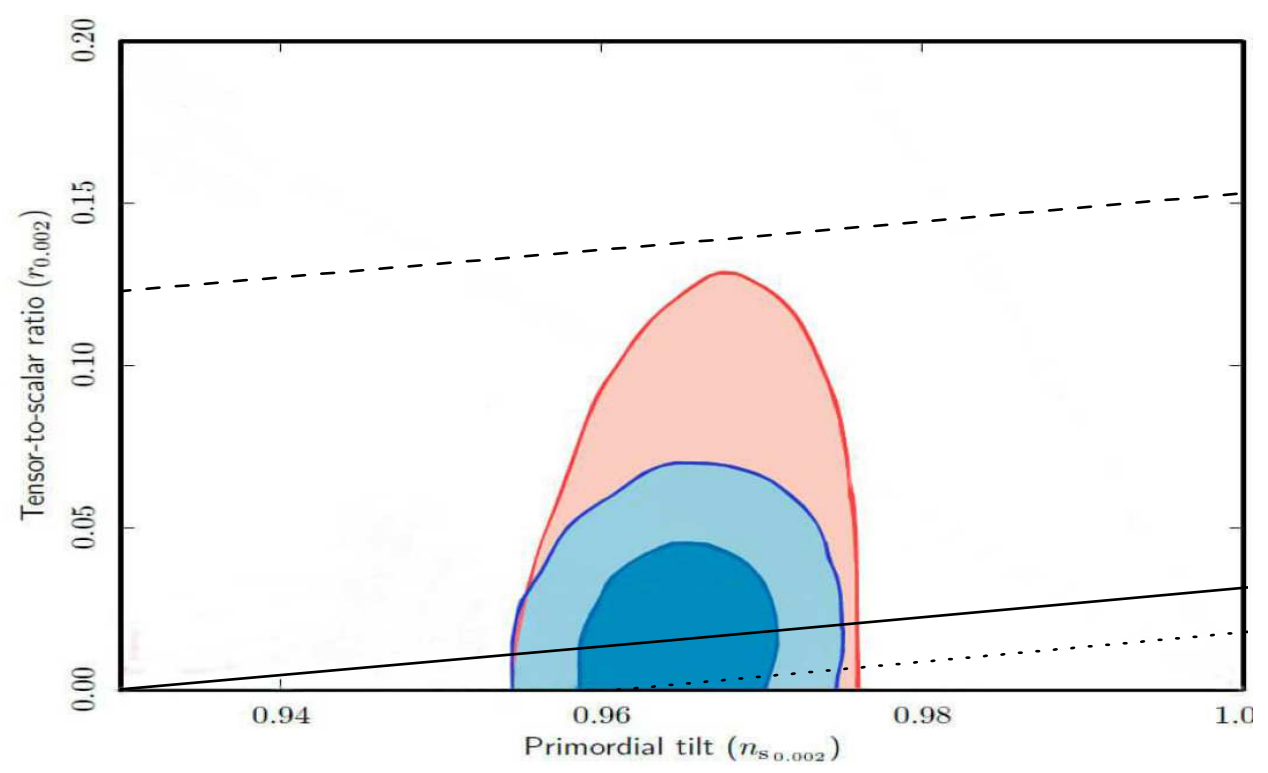

FIG. 1: The panel shows the tensor-to-scalar ratio $r$ as a function of the scalar spectral index $n_{s}$, for three different values of the parameter $A_{1}^{t}$. In this panel we have considered the twomarginalized constraints joint $68 \%$ and $95 \% \mathrm{CL}$ at $k=0.002 \mathrm{Mpc}^{-1}$ from the Planck 2018 results [31]. In the plot the dashed, solid and dotted lines correspond to the values of parameter $A_{1}^{t}=0.99$, $A_{1}^{t}=0.999$ and $A_{1}^{t}=0.1$, respectively.

$r=r\left(n_{s}\right)$ (at $68 \%$ and $95 \% \mathrm{CL}$ at $k=0.002 \mathrm{Mpc}^{-1}$ ) from the new Planck results[31]. By considering Eqs.(43), (152) and (153), we numerically obtain the parametric plot for tensor to scalar ratio in terms of the scalar spectral index or the consistency relation $r=r\left(n_{s}\right)$ for three values of the parameter $A_{1}^{t}$ (integration constant, see Eq.(35)). In this plot, we have considered that the dashed, solid and dotted lines correspond to the values $A_{1}^{t}=0.99$, $A_{1}^{t}=0.999$ and $A_{1}^{t}=0.1$, respectively. On the other hand, from Eqs.(52) and (53) we can note that the scalar power spectrum $\mathcal{P}_{\mathcal{R}}$ depends of the parameters $\mathcal{P}_{\mathcal{R}}=\mathcal{P}_{\mathcal{R}}\left(k, \beta, C_{0}^{t}, A_{1}^{t}\right)$ and the scalar spectral index $n_{s}=n_{s}\left(k, \beta, C_{0}^{t}\right)$. In this way, we numerically find from these relations that the values of the parameters $\beta=-1.35997$ (or $\beta_{1}=1.85997$ since $\beta_{1}=1 / 2-\beta$ ) and $C_{0}^{t}=3.947 \times 10^{-3}$ (or $\alpha_{1} \simeq 0.063$ since $\left.\alpha_{1}=\sqrt{C_{0}^{t}}\right)$ correspond to the parameter $A_{1}^{t}=0.1$ (dotted line). Here we have used the values of $\mathcal{P}_{\mathcal{R}}=2.2 \times 10^{-9}$ and $n_{s}=0.964$ at $k=0.002 \mathrm{Mpc}^{-1}$. Also, we find that the tensor to scalar ratio for these values at $k=0.002 \mathrm{Mpc}^{-1}$ is given by $r(k=0.002)=0.0015$. Analogously, we numerically find that for the value $A_{1}^{t}=0.999$, we have the parameters $\beta=-1.35998$ and $C_{0}^{t}=3.963 \times 10^{-3}$ (solid line). Here we obtain that the tensor to scalar ratio at $k=0.002 \mathrm{Mpc}^{-1}$ corresponds to 
$r(k=0.002)=0.0016$. For the situation in which $A_{1}^{t}=0.99$ (dashed line), we numerically find the values of the parameters $\beta=-1.3601, C_{0}^{t}=4.110 \times 10^{-3}$ and the ratio $r(k=$ $0.002)=0.138$. Here, we have considered that the initial conformal time $\left|\eta_{i}\right|=400.000$, since the tensor to scalar ratio $r$ depends of $r=r\left(k, \beta, C_{0}^{t}, A_{1}^{t}, \eta_{i}\right)$. In this form, from Fig:1 we note that the value of the integration constant $A_{1}^{t} \simeq 0.1$ since it is well corroborated by Planck 2018 results. On the other hand, for values of the constant $A_{1}^{t}<0.1$, we find that the ratio $r>0.1$ and then the model II is disfavored from Planck data. Also, we mention that for values of the integration constant $A_{1}^{t}>0.1$ the tensor to scalar ratio $r$ becomes negative and the model is excluded from observational data. Thus, from Fig.1, we observe that for the values of the parameters $A_{1}^{t} \simeq 0.1, \beta \simeq-1.4$ and $C_{0}^{t} \simeq 10^{-3}$, the model II describe for the ansatz given by Eq.(32), is well corroborated by Planck 2018 data, however we note that the parameter-space is very small. Also, we note that from Eqs.(47) and (48), we can obtain the values $C_{0}^{S} \simeq 0.007, C_{1}^{S} \simeq-0.2$ and $C_{2}^{S} \simeq 1.6$, respectively. This suggests that parameter-space for our model II is similar to de Sitter Universe in which $C_{0}^{S}=0, C_{1}^{S}=0$ and $C_{2}^{S}=2$.

On the other hand, from Eqs.(17), (33) and (35) we find that the arbitrary function $F$ in terms of the time $\eta$ can be written as

$$
F(\eta)=\frac{z_{t}^{2}}{a^{2}}=a^{-2} A_{1}^{t 2} W_{\alpha_{t}, \beta}(\eta)^{2} \propto a^{-2}|\eta|^{2 \beta_{1}} e^{-2 \alpha_{1}|\eta|} .
$$

Here we have considered the large scale limit of the Whittaker function $W_{\alpha_{t}, \beta}$.

In order to build the effective potential, we find that combining Eqs.(6) and (17), the potential can be rewritten as function of the variable $z_{t}$ as

$$
V=a^{-4}\left\{z_{t}^{\prime 2}+z_{t} z_{t}^{\prime \prime}\right\}
$$

In this way, we find the effective potential in terms of the conformal time $\eta$ results

$$
V(\eta)=\frac{F}{a^{2}}\left[2 x^{2}-(\mathcal{H}+x)^{2}-\frac{\beta_{1}}{\eta^{2}}\right],
$$

where the quantity $x$ is defined as $x=\frac{\beta_{1}}{|\eta|}-\alpha_{1}$ and the function $F$ is given by Eq.(54). Here we have also used that $F^{\prime}=2 F[x-\mathcal{H}]$.

From Eq.(44) (or equivalently of Eq.(10) ) we obtain that the relation between the scalar field and the conformal time can be found from the differential equation given by

$$
\varphi^{\prime 2}(\eta)=\frac{2 F}{\omega}\left[x^{2}-3(x-\mathcal{H})^{2}+\frac{\beta_{1}}{\eta^{2}}\right] .
$$


On the other hand, in order to reconstruct the background, in particular the effective potential $V(\phi)$ and the scale factor $a(\eta)$, we will consider a simple example in which the coupling function $F(\varphi) \propto \varphi^{2}=B_{0} \varphi^{2}$ and also the parameter $\omega=$ const. In this case, we have that $F^{\prime}=2 B_{0} \varphi \varphi^{\prime}=2 F(x-\mathcal{H})=2 B_{0} \varphi^{2}(x-\mathcal{H})$ and combining with Eq. (57) we get

$$
\frac{\varphi^{\prime}}{\varphi}=D_{0}\left[x^{2}+\frac{\beta_{1}}{\eta^{2}}\right]^{1 / 2}, \text { where } D_{0}=\sqrt{\frac{2 B_{0}}{6 B_{0}+\omega}} .
$$

Here we have considered the positive sign for simplicity. In this form, we find that the solution of the Eq.(58) can be written as

$$
\varphi(\eta)=\varphi_{0} e^{D_{0} y}\left(\frac{|\eta|}{2 b_{1}+b_{2}|\eta|+2 \sqrt{b_{1}} y}\right)^{D_{0} \sqrt{b_{1}}}\left(b_{2}+2|\eta| b_{3}+2 \sqrt{b_{3}} y\right)^{-D_{0} \beta_{1}}
$$

where the quantity $y$ is defined as $y=y(\eta)=\sqrt{\left.b_{1}+|\eta| b_{2}+\eta^{2} b_{3}\right)}, \varphi_{0}$ denotes an integration constant and the constants $b_{1}, b_{2}$ and $b_{3}$ are defined as

$$
b_{1}=\beta_{1}\left(\beta_{1}+1\right), \quad b_{2}=-2 \beta_{1} \alpha_{1}, \quad b_{3}=\alpha_{1}^{2},
$$

respectively.

By combining Eqs.(54) and (59) we obtain that the reconstruction for the scale factor as a function of the conformal time $\eta$ is given by

$$
a(\eta) \propto e^{-\left(\alpha_{1}|\eta|+D_{0} y\right)}\left(2 b_{1}+b_{2}|\eta|+2 \sqrt{b_{1}} y\right)^{D_{0} \sqrt{b_{1}}}\left(b_{2}+2|\eta| b_{3}+2 \sqrt{b_{3}} y\right)^{D_{0} \beta_{1}}|\eta|^{\beta_{1}-D_{0} \sqrt{b_{1}}}
$$

In Fig,2, we show the evolution of the scale factor $a(\eta)$ panel) and its acceleration $a^{\prime \prime}(\eta)$ versus the conformal time $\eta$ (left panel). In the right panel we show the effective potential in terms of the scalar field $\varphi$. In these panels, we have used the parameters $D_{0}=1$, $C_{0}^{t}=3.947 \times 10^{-3}$ and $\beta=-1.35997$, respectively.

For the plot of the scale factor in the left panel, we consider the analytical reconstruction given by Eq.(60) for $a(\eta)$ (see solid line) and checking its acceleration $a^{\prime \prime}(\eta)>0$ (dashed line). On the other hand, in order to build the effective potential as a function of the scalar field i.e., $V(\varphi)$, we have considered Eq.(56) and the solution $\varphi(\eta)$ given by Eq.(59). Thus, we can reconstruct the effective potential as a function of the scalar field $\varphi$ considering a parametric plot from $V(\eta)$ and $\varphi(\eta)$, respectively (see right panel). 

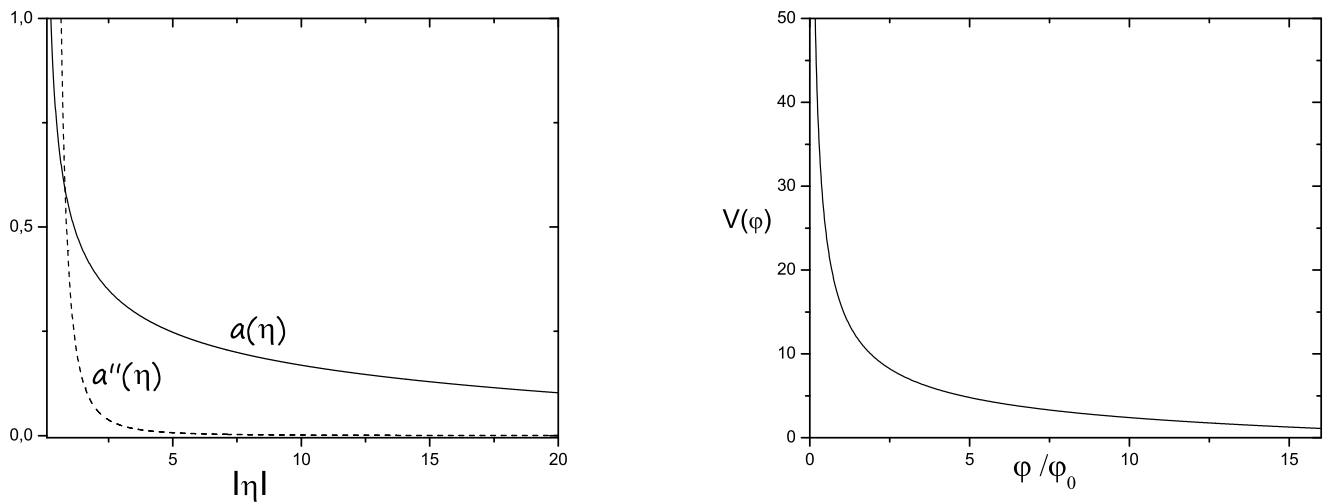

FIG. 2: In the left panel we show the scale factor $a(\eta)$ (solid line) and its acceleration $a^{\prime \prime}(\eta)$ (dashed line) as a function of the conformal time $\eta$. In the right panel we show the effective potential $V(\varphi)$ in terms of the scalar field. In both panels we have considered $D_{0}=1, C_{0}^{t}=3.947 \times 10^{-3}$ and $\beta=-1.35997$.

\section{CONCLUSIONS}

In this article, we have studied an approach to exact solutions of cosmological perturbations in the context of a generalized scalar tensor theory for two models characterized for the relation between the variables of the modes of the tensor $\left(z_{t}\right)$ and scalar $\left(z_{s}\right)$ perturbations, respectively.

For both models, we have obtained analytical solutions for the corresponding power spectrum of the curvature perturbations, scalar spectral index, the power spectrum of the tensor perturbations, and the tensor to scalar ratio together with the background variables.

For the model I we have considered that the relation between the modes is given by $z_{s} \propto z_{t}$. For this model we have found a particular solution in which $z_{s} \propto z_{t} \propto \eta^{q}$, where the parameter $q$ has relation with the proportionality constant. For this model we have obtained that this model is not well supported by the Planck data, since the tensor to scalar ratio $r>0.1$. This suggests that the model $\mathrm{I}$ in which $z_{s} \propto z_{t}$ does not work. However, although the model $z_{s} \propto z_{t}$ is not well agreement from the observational data, we have considered an example to reconstruct the background variables (scalar potential $V(\varphi)$ and coupling function $F(\varphi)$ ), by assuming the power-law inflation and the parameter $\omega=1$. In this context, we have found that the coupling function $F(\varphi)$ is given by $F(\varphi) \propto \varphi^{2}$ and the effective potential corresponds to the power-law potential in which $V(\varphi) \propto \varphi^{2 \beta}$ (see 
Eq.(28) $)$.

For the model II we have assumed a more general relation between the variables $z_{s}$ and $z_{t}$ given by Eq.(29). In order to obtain analytical solution to the cosmological perturbations, we have considered an interesting ansatz-function $f(\eta)$ given by Eq.(32). From this ansatz on $f(\eta)$, we have found that the Fourier modes of the scalar and tensor perturbations can be written in terms of the Whittaker functions. By assuming the large and small limits for each one of the Fourier modes, we have found analytical expressions for the scalar and tensor power spectral as a function of the wave number $k$. From these expressions for the amplitudes, we have obtained the constraints on the different parameters considering the new Planck 2018 results. In this context, we have used the consistency relation $r=r\left(n_{s}\right)$, and we have noted that our parameter-space is very reduced. In this sense, we have observed from the plane-trajectories $r=r\left(n_{s}\right)$ the model II is well supported by Planck results only if

the parameters in our model are $C_{0}^{S} \simeq 0.007, C_{1}^{S} \simeq-0.2$ and $C_{2}^{S} \simeq 1.6$, respectively. Thus, we have observed that parameter-space for our model II is similar to de Sitter inflation in which $C_{0}^{S}=0, C_{1}^{S}=0$ and $C_{2}^{S}=2$. In relation to the reconstruction of the background variables, for simplicity we have given a particular coupling function $F(\varphi) \propto \varphi^{2}$, with which we have found the reconstruction for the scale factor $a(\eta)$ and the effective potential $V(\varphi)$, respectively (see Fig.(2) ).

Finally in this article, we have not addressed the reconstruction of the background variables and cosmological perturbations to another ratios $z_{s} / z_{t}$ in terms of the conformal time, see Eq.(31). We hope to return to this point in the near future.

\section{Acknowledgments}

J.A.B. was supported by COMISION NACIONAL DE CIENCIAS Y TECNOLOGIA through FONDECYT Grant $\mathrm{N}_{0}$ 11170083. C.G. was supported by Proyecto DIUDA REG-

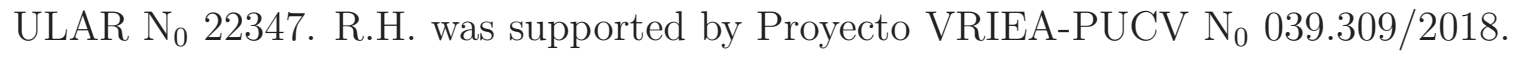

[1] A.A. Starobinsky, Phys. Lett. B 91, 99 (1980); A. Guth, Phys. Rev. D 23, 347 (1981).

[2] A.D. Linde, Phys. Lett. B 108, 389 (1982). 
[3] D. Larson et al., Astrophys. J. Suppl. 192, 16 (2011); C. L. Bennett et al., Astrophys. J. Suppl. 192, 17 (2011).

[4] P. A. R. Ade et al. [Planck Collaboration], Astron. Astrophys. 594, A20 (2016).

[5] S. W. Hawking,Phys. Lett. B 115, 295 (1982);A. A. Starobinsky, Phys. Lett. B 117, 175 (1982); J.M. Bardeen, P.J. Steinhardt and M.S. Turner, Phys. Rev.D 28, 679 (1983).

[6] P. Jordan, Z. Phys. 157, 112 (1959).

[7] C. Brans and R.H. Dicke, Phys. Rev. 124, 925 (1961).

[8] P. G. Bergmann Int. J. Theor. Phys. 1, 25-36 (1968); R. V. Wagoner Phys. Rev. D 1, 3209-16 (1970).

[9] N.A. Chernikov, E.A. Tagirov, Quantum theory of scalar field in de Sitter space. Ann. Inst. H. Poincare A 9, 109171411 (1968).

[10] T. Clifton, P. G. Ferreira, A. Padilla and C. Skordis, Phys. Rept. 513, 1 (2012).

[11] A. Zee, Phys. Rev. Lett. 44, 703 (1980); F. Cooper, G. Venturi, Phys. Rev. D 24, 3338 (1981); C. Wetterich, Nucl. Phys. B 302, 668 (1988).

[12] B.L. Spokoiny, Phys. Lett. B 147, 39 (1984); R. Fakir, W.G. Unruh, Astrophys. J 394, 396 (1992); V. Faraoni, E. Gunzig, P. Nardone, Fund. Cosmic Phys. 20, 121 (1999); E. Komatsu, T. Futamase, Phys. Rev. D 59, 064029 (1999).

[13] T. Damour and K. Nordtvedt, Phys. Rev. Lett. 70, 2217 (1993); J. Garcia-Bellido and D. Wands, Phys. Rev. D 52, 6739 (1995); J. Martin, C. Ringeval, and V. Vennin, Phys. Dark Univ. 5-6, 75 -235 (2014).

[14] L. Jarv, K. Kannike, L. Marzola, A. Racioppi, M. Raidal, M. Runkla, M. Saal and H. Veermae, Phys. Rev. Lett. 118, no. 15, 151302 (2017).

[15] D. I. Kaiser, Phys. Rev. D 52, 4295 (1995).

[16] V. Faraoni, Phys. Rev. D 53, 6813 (1996); V. Faraoni, Fundam. Theor. Phys. 139 (2004).

[17] J. A. Casas, J. Garcia-Bellido and M. Quiros, Class. Quant. Grav. 9, 1371 (1992);A. M. Green and A. R. Liddle, Phys. Rev. D 54, 2557 (1996); X. l. Chen and M. Kamionkowski, Phys. Rev. D 60, 104036 (1999); C. Campuzano, S. del Campo and R. Herrera, JCAP 0606, 017 (2006); S. Carloni, S. Capozziello, J. A. Leach and P. K. S. Dunsby, Class. Quant. Grav. 25, 035008 (2008); S. del Campo, R. Herrera and P. Labrana, JCAP 0907, 006 (2009).

[18] J. Garcia-Bellido and A. D. Linde, Phys. Rev. D 52, 6730 (1995); J. Garcia-Bellido and D. Wands, Phys. Rev. D 52, 5636 (1995); D. F. Torres, Phys. Lett. A 225, 13 (1997); S. Tsu- 
jikawa, Phys. Rev. D 62, 043512 (2000); A. A. Starobinsky, S. Tsujikawa and J. Yokoyama, Nucl. Phys. B 610, 383 (2001); R. Nagata, T. Chiba and N. Sugiyama, Phys. Rev. D 69, 083512 (2004); D. F. Torres, Phys. Lett. A 225, 13 (1997); S. del Campo, C. Gonzalez and R. Herrera, Astrophys. Space Sci. 358, no. 2, 31 (2015); C. Gonzalez and R. Herrera, Eur. Phys. J. C 77, no. 9, 648 (2017).

[19] V. Lukash, Pis'ma Zh. Eksp. Teor. Fiz. 49, 631 (1980); G. Chibisov and V. Mukhanov, Galaxy and phonons P.N. Lebedev Phys. Inst. 162 (1980); G. Chibisov and V. Mukhanov, Mon. Not. R. Astron. Soc. 200, 535 (1982).

[20] F. Lucchin and S. Matarrese, Phys. Rev. D 32, 1316 (1985).

[21] D. H. Lyth and E. D. Stewart, Phys. Lett. B 274, 168 (1992); E. D. Stewart and D. H. Lyth, Phys. Lett. B 302, 171 (1993).

[22] V. F. Mukhanov, H. A. Feldman, and R. H. Brandenbarger, Phys. Rep. 215, 203 (1992).

[23] R. Easther, Class. Quantum Grav. 13, 1775 (1996).

[24] J. Martin, D. Schwarz, Phys.Lett.B 500, 1-7 (2001).

[25] R. Herrera and R. G. Perez, Phys. Rev. D 93, no. 6, 063516 (2016).

[26] I. J. Grivell and A. R. Liddle, Phys. Rev. D 54, 7191 (1996); A. Makarov, Phys. Rev. D 72, $083517(2005)$.

[27] A. A. Starobinsky, JETP Lett. 82, 169 (2005); [Pisma Zh. Eksp. Teor. Fiz. 82, 187 (2005)].

[28] B. Boisseau, G. Esposito-Farese, D. Polarski and A. A. Starobinsky, Phys. Rev. Lett. 85, 2236 (2000)

[29] J. O. Gong and E. D. Stewart, Phys. Lett. B 510, 1 (2001); E. D. Stewart, Phys. Rev. D 65, 103508 (2002); J. Choe, J. O. Gong and E. D. Stewart, JCAP 0407, 012 (2004).

[30] H. Wei, R. G. Cai and A. Wang, Phys. Lett. B 603, 95 (2004)

[31] Y. Akrami et al. [Planck Collaboration], arXiv:1807.06211 [astro-ph.CO].

[32] P. G. Bergmann, Int. J. Theor. Phys 1 , 25 (1968); K. Nordtvedt, Astrophys. J 161 , 1059 (1970); R. Wagoner, Phys. Rev. D 1 , 3209 (1970).

[33] J. Bardeen, Phys. Rev. D 22, 1882 (1980).

[34] H.Noh and J.Hwang, Phys.Lett. B515, 231-237 (2001).

[35] S.Tsujikawa and B.Gumjudpai, Phys.Rev.D69, 123523 (2004).

[36] P. A. R. Ade et al. [BICEP2 and Keck Array Collaborations], Phys. Rev. Lett. 116, 031302 (2016). 
[37] D. Kaiser, Phys. Rev. D 81, 084044 (2010).

[38] B. L. Spokoiny, Phys. Lett. B 147, 39 (1984); F. S. Accetta, D. J. Zoller, and M. S. Turner, Phys. Rev. D 31, 3046 (1985);

[39] R. Fakir and W. G. Unruh, Phys. Rev. D 41, 1792 (1990); D. I. Kaiser, Phys. Rev. D 49, 6347 (1994); J. L. Cervantes-Cota and H. Dehnen, Phys. Rev. D 51, 395 (1995).

[40] Abramowitz, M. and Stegun, I. A. (Eds.). Handbook of Mathematical Functions with Formulas, Graphs, and Mathematical Tables, 9th printing. New York: Dover, (1972).

[41] S. Tsujikawa, "Introductory review of cosmic inflation," hep-ph/0304257. 